\title{
Literaturbericht
}

\author{
Timo Grunden
}

\section{Der Bundespräsident: Monarchisches Artefakt oder politisches Verfassungsorgan?*}

\section{Der Bundespräsident: Zwei Interpretationsparadigmen}

„Warum gibt es in einem parlamentarischen Regierungssystem einer demokratischen Republik überhaupt einen Staatspräsidenten?“ Heinz Rausch (1984: 14) war nicht der Erste, der sich diese Frage stellte. Bereits im Verfassungskonvent von Herrenchiemsee wurde die Notwendigkeit eines solchen Amtes bezweifelt. Der Sozialdemokrat Hermann Brill nannte es ein entbehrliches „Requisit“ (zit. nach Niclauß 1998: 186) und auch andere Verfassungsväter äußerten Bedenken, wenngleich sie diese nicht in erster Linie verfassungstheoretisch, sondern mit dem Verweis auf die Besatzungsmacht der Siegermächte und den provisorischen Charakter des Grundgesetzes begründeten. ${ }^{1}$ Es war zwar nur eine kleine Minderheit, die ein Staatsoberhaupt weder für erforderlich noch für wünschenswert erachtete. Eine Minderheit waren aber eben auch diejenigen, die sich seiner konstitutionellen Bedeutung in der Verfassungsrealität der neuen Republik von Anfang an absolut sicher waren. Gewissheit gibt es bis heute nicht. Weil „Stellung und Aufgaben des Bundespräsidenten $[\ldots]$ nur ansatzweise vom Grundgesetz bezeichnet" sind (Nettesheim 2005: 1034), bietet das Amt seit jeher viel Raum für ausladende Verfassungsexegesen und politikwissenschaftliche Funktionsbestimmungen.

Die verschiedenen Perspektiven auf das Amt des Bundespräsidenten sollen im Folgenden durch die Unterscheidung von zwei Interpretationsparadigmen systematisiert werden: Die in der Politikwissenschaft dominierende und in Anlehnung an Marcus Höreth $(2008,2012)$ als Westminster-Paradigma rekonstruierte Per-

* Eine ausführlichere Diskussion der Literatur zum Bundespräsidenten findet sich bei Grunden 2013, ebenso auch Überlegungen zur Pfadabhängigkeit des Amtes sowie zu Möglichkeiten und Grenzen einer politisierten Amtsführung.

1 Zur Genese des Amtes des Bundespräsidenten im Konvent von Herrenchiemsee und im Parlamentarischen Rat vergleiche Niclauß 1998: 176-202. 
spektive wird dem Gewaltenteilungsparadigma gegenübergestellt, das vor allem durch die Staatsrechtswissenschaften geprägt worden ist, aber zunehmend auch in der Politikwissenschaft rezipiert wird. Im Westminster-Paradigma wird die konstitutionelle Bedeutung des Bundespräsidenten weniger durch die Verfassung als durch die normativ-funktionale Logik des parlamentarischen Regierungssystems definiert. Demnach ist der Bundespräsident ein republikanischer Monarch, dessen Rolle angesichts der ,Funktionslogik“ der parlamentarischen Demokratie vor allem daran festgemacht wird, was er ungeachtet seiner verfassungsrechtlichen Möglichkeiten nicht tun sollte: nämlich mit Worten oder gar konstitutionellen Handlungen in die reziproke Legitimationskette Volk $>$ Parlament $>$ Exekutive eingreifen und den Dualismus von Regierung und Opposition stören (Abschnitt 2).

Dagegen ist die Staatsrechtswissenschaft für normativ-funktionale Argumente weniger empfänglich, zumal sie naturgemäß den Verfassungstext nicht einfach den empirischen Kausalmechanismen eines Regierungssystems unterordnen kann. Dementsprechend kreist die staatsrechtliche Literatur über den Bundespräsidenten weniger um die Frage, wie er sein Amt ausüben sollte, sondern welche Macht der Amtsinhaber angesichts seiner institutionellen Kompetenzen ausüben könnte - ein Ansatz, der zunehmend auch in der Politikwissenschaft auf Interesse stößt. Dem Staatsoberhaupt wird zwar auch hier keine konstitutionelle Machtposition zugestanden, die annähernd an jene im Semi-Präsidentialismus heranreichen würde. Aber er wird im Hauptstrom der entsprechenden Literatur auch nicht außerhalb des „politischen Kräftespiels“ verortet, sondern aufgrund seiner konstitutionellen Potenziale in den Rang einer eigenständigen Gewalt im Mobile sich gegenseitig kontrollierender Verfassungsorgane erhoben (Abschnitt 3).

Der bisherigen Auseinandersetzung der Staatswissenschaften mit dem Amt und der Amtsführung der Bundespräsidenten gelten die letzen Überlegungen dieses Beitrags. Der folgende Überblick wird zeigen, dass sich die einschlägige Literatur vor allem der Grammatik des Imperativs und des Konjunktivs bedient, indem sie danach fragt, ,was er kann, darf und muss bzw. könnte, dürfte und müsste“ (Strohmeier 2008). Wovon es zu wenig gibt, ist der Indikativ: eine nicht nur durch Plausibilitätsargumentationen gestützte äußere Anschauung, sondern eine profunde empirische Analyse dessen, was die Bundespräsidenten tatsächlich tun und wie sie wirken (Abschnitt 4). 
Der Bundespräsident: Monarchisches Artefakt oder politisches Verfassungsorgan?

\section{Wie das Amt ausgeübt werden sollte: Das Westminster-Paradigma}

Blickt man durch die „Westminster-Brille“ (Höreth 2012: 2) auf das Amt des republikanischen Staatsoberhauptes, erkennt man nicht viel mehr als ein institutionelles Artefakt aus der Evolution der Verfassungsgeschichte. „Im Institutionengefüge demokratischer Verfassungsstaaten", so Hans-Peter Schwarz (2012: 299), ist es ein „Fremdkörper“, in dem sich ,in republikanischer Gewandung eine Fortentwicklung der Monarchie“ zeige. Akzeptiert man diese Deutung des Amtes, dann scheint auch die Frage nach der Rolle und Funktion des Staatspräsidenten im parlamentarischen Regierungssystem gar nicht mehr die entscheidende zu sein. Umso wichtiger wird vielmehr jene nach der Rolle und den Funktionen, die die republikanischen Nachfolger des Monarchen im Zeitverlauf verloren haben. Dementsprechend wird das Amt des Bundespräsidenten in der politikwissenschaftlichen Regierungslehre in erster Linie über Negativlisten definiert. Was bleibt, ist eine restriktiv zu handhabende und der Konnotation nach auch eher vernachlässigbare „Reservefunktion“ für den „Krisenfall“ sowie eine oft nur diffus umschriebene „Symbol- und Integrationsfunktion“ “im „Normalfall“. Angesichts des Verfassungstextes und seiner Genese ist diese Darstellung des Amtes durchaus konsequent. Schließlich rührt die „Unschärfe“, die Martin Nettesheim (2005: 1034) dem Grundgesetz im Hinblick auf den Bundespräsidenten attestiert, daher, dass die Verfassungsgeber vor allem anderen definieren wollten, was der Bundespräsident der Bundesrepublik Deutschland nicht ist: eine exekutive Gewalt, die in der Lage wäre, dem Volk und seinem Parlament die Souveränität zu entreißen, um sie in die Hände von Totalitaristen zu legen. Die Erinnerung an die fatale Rolle, die Reichspräsident Hindenburg bei der Zerstörung der Weimarer Demokratie spielte, habe im Parlamentarischen Rat, so Werner Patzelt (2005: 295), dermaßen ,die Debatten über die Stellung des Bundespräsidenten geprägt, dass schließlich nur noch recht unklar herausgearbeitet wurde, was eigentlich positiv die Aufgabe des Bundespräsidenten als Staatsoberhaupt sei.“

Der Bundespräsident erhielt überwiegend notarielle Pflichtaufgaben, bei deren Ausübung ihm kein eigener Handlungsspielraum zusteht. Die wenigen seiner politischen Entscheidungskompetenzen sind in ihrer Mehrzahl auf die Bewältigung von 
Regierungskrisen zugeschnitten. ${ }^{2}$ Andererseits: Wenn die Mitglieder des Parlamentarischen Rates wirklich eine konstitutionelle Monarchie vor Augen hatten, als sie das Amt mit Kompetenzen und Aufgaben ausstatteten, wie Karlheinz Niclauß (1998: 189) mit Verweis auf Volker Otto (1971) bemerkt, dann sind dies noch immer erstaunlich viele. Und im Vergleich etwa zur englischen oder niederländischen Krone sind sowohl die Kompetenzen als auch die nicht-regulierten öffentlichen Wirkungsmöglichkeiten des deutschen Bundespräsidenten doch recht großzügig bemessen.

Und dennoch ist es das Westminster-Paradigma, dessen Prämissen die Funktionsbestimmungen des Amtes über Jahrzehnte anleiteten und das dem Bundespräsidenten zwar keine unpolitische, aber doch entpolitisierte Rolle von allenfalls geringer institutioneller Bedeutung zuweist. Das theoretische Fundament für alle Ansätze, die das Bundespräsidentenamt entlang normativer Funktionen im parlamentarischen Regierungssystem analysieren, legte Werner Kaltefleiter in seiner 1970 erschienenen Habilitationsschrift. Sie verdient es, etwas ausführlicher referiert zu werden, weil man ohne Übertreibung sagen kann, dass seine Interpretation des Amtes zur "herrschenden Meinung“ in der Politikwissenschaft avancierte. Kaltefleiter vergleicht die institutionelle Stellung und die Funktionen von Staatsoberhäuptern in parlamentarischen Regierungssystemen, um der Frage nachzugehen, „wie das Amt des Staatsoberhauptes beschaffen sein $m u \beta$, damit es der Arbeitsfähigkeit des parlamentarischen Regierungssystems dient" (Kaltefleiter 1970: 18). Im Mittelpunkt steht der „systemnormative Aspekt" des Amtes: Weniger der geschriebene Verfassungstext als die Struktur des parlamentarischen Regierungssystems bestimme die Funktion einzelner Institutionen (ebd.: 23). Dazu zählen auch das ,latente und manifeste Normensystem und die Verhaltenserwartungen der verschiedenen politischen Gruppen“ zum relevanten Teil der Verfassung in einem „umfassenden Sinne" (ebd.: 15).

Um eine dem parlamentarischen Regierungssystem angemessene Funktionsdefinition des Staatsoberhauptes und entsprechende Kriterien einer angemessenen

2 Als da wären: das Recht, den Bundestag einzuberufen (Art. 39 GG); das Vorschlagsrecht zur Wahl des Bundeskanzlers im ersten Wahlgang; das Recht, eine Minderheitsregierung nach dem dritten Wahlgang zu ernennen oder den Bundestag aufzulösen (Art. 63 GG); das Recht zur Auflösung des Bundestages, wenn eine Vertrauensfrage des Kanzlers scheitert und dieser eine Auflösung vorschlägt bzw. das Recht, die gescheiterte Regierung als Minderheitskabinett im Amt zu lassen (Art. 68 GG); die Erklärung des Gesetzgebungsnotstandes auf Antrag der Bundesregierung und mit Zustimmung des Bundesrates (Art. 81 GG); die Prüfung von Gesetzen auf ihre Verfassungsmäßigkeit (Art. 82 GG); das Begnadigungsrecht (Art. 60 GG) und schließlich das Recht zur Anrufung des Bundesverfassungsgerichts bei Organstreitigkeiten (Art. 93 GG). 
Der Bundespräsident: Monarchisches Artefakt oder politisches Verfassungsorgan?

Amtsführung zu entwickeln, nutzt Kaltefleiter das „Klassisch-ParlamentarischeRegierungssystem“ (1970) Englands als idealtypische Referenz. Etwaigen Einwänden gegen diesen Ansatz begegnet er mit dem Argument, die Unterschiede zwischen institutioneller Monarchie und parlamentarischer Republik beständen lediglich in den „Selektionssystemen“ (Erbfolge vs. Wahl), nicht aber in den Funktionen des Staatsoberhauptes (ebd.: 23 f.). In beiden Verfassungssystemen ist es die Reserveund Symbolfunktion, die ihm eine konstitutionelle Bedeutung verleihen. Somit rechtfertigt sich auch die Existenz des Bundespräsidentenamtes dadurch, dass er in der außerordentlichen Situation einer Regierungskrise willens und fähig ist, die Wiederherstellung einer stabilen und handlungsfähigen Regierung herbeizuführen. Er hat dabei aber keine eigenen (partei-)politischen Ziele zu verfolgen, sondern einzig und allein ,den gestörten Mechanismus des parlamentarischen Systems in Ordnung zu setzen" (ebd.: 33). Im Normalfall symbolisiert das Staatsoberhaupt durch die „Verkündung der von Regierung und Parlament beschlossenen und verantworteten Gesetze“ die „Einheit der Nation“. Die Minderheit hat die Entscheidungen der Mehrheit zu akzeptieren; ,sie gelten für alle“ (ebd.: 32).

Die Symbolfunktion ist bei Kaltefleiter keine eigenständige Kategorie; sie ist Teil der Reservefunktion und verlangt die gleiche strikte Überparteilichkeit. Präsident oder Monarch haben zwar das Recht, ihre Meinung zu äußern, ,zu warnen und zu mahnen", aber sie sollten das nicht öffentlich tun. Ihre Symbolfunktion beschränkt sie hier auf die „Artikulation von allem Unbestrittenen, des Verfassungskonsenses im weitesten Sinne" (Kaltefleiter 1970: 32). Halten sie sich nicht an dieses Gebot, drohen sie in den politischen Wettbewerb abzurutschen, wo sie über kurz oder lang ihre wichtigste Handlungsressource verlieren werden: ihre Autorität. Ohne Autorität aber, die vor allem anderen auf dem Nimbus ihrer Überparteilichkeit beruht, werden sie im Krisenfall ihrer Reservefunktion nicht entsprechen können. „Daraus folgt, daß öffentliche Reden und Ausführungen des Staatsoberhauptes entweder nur unstrittige Fragen betreffen oder strittige Fragen nur unentschieden behandeln können oder mit der amtierenden Regierung abgesprochen sein müssen" (ebd.: 58). Das Grundgesetz lege vielleicht eine ,expansivere Auslegung der Funktionen“ des Präsidenten nahe, aber eine solche Interpretation stünde ,nicht im Einklang mit den funktionalen Erfordernissen des parlamentarischen Regierungssystems" (ebd.: 275). Die „verfassungsrechtlich begründeten Entscheidungsspielräume des Bundespräsidenten“ sind letztlich nur von „theoretischer Natur". Eine angemessene Amtsführung zeichne sich gerade dadurch aus, dass der Amtsinhaber den Rahmen des Verfassungsrechts nicht ausschöpft (ebd.: 276).

In der Mehrzahl der Überblicksdarstellungen des politischen Systems der Bundesrepublik ist der Einfluss Kaltefleiters nicht zu überlesen. Allerdings ist auch ein 
bedeutender Unterschied auffällig: Die Symbolfunktion wird von der Reservefunktion getrennt und um Integrations- und Repräsentationsaspekte erweitert. Klarer wird das Aufgaben- und Funktionsprofil des Amtes dadurch nicht. Zudem: Wer hier nach einer Antwort auf die Eingangsfrage sucht, wird sich im Verlauf der Lektüre eines Gefühls der Ernüchterung nicht erwehren können.

Zunächst ist mit Stefan Marschall festzustellen, dass „,der Präsident in der wissenschaftlichen Beachtung vergleichsweise ein Schattendasein [fristet]“. Ihm werden ,üblicherweise nur wenige Seiten gegönnt“ (2007: 176). Manfred G. Schmidt (2007) etwa widmet ihm ganze drei Textseiten. Er verweist kurz auf die notariellen Funktionen und die „Reservegewalt“ des Bundespräsidenten, stellt dann aber klar: „Normalerweise liegt die politische Bedeutung des Amtes des Bundespräsidenten jedoch hauptsächlich in stilgebenden, repräsentativen, zeremoniellen Funktionen“ (177). Auch das von Ernst Benda, Werner Maihofer und Hans-Jochen Vogel herausgegebene „Handbuch des Verfassungsrechts“ (1983), um ein Beispiel aus der Staatsrechtslehre anzuführen, erwähnt den Bundespräsidenten auf gerade mal neun von 1.417 Seiten.

Kurt Sontheimer, der sich ebenfalls nicht lange mit dem Bundespräsidenten aufhält, bringt den Grund für das geringe Interesse auf den Punkt: Die Stellung des Bundespräsidenten entspräche ,im großen und ganzen“ der Funktion, „die der Monarch im britischen Regierungssystem wahrnimmt“ (1995: 279). Das „Bonner Grundgesetz“, schreibt Sontheimer weiter, „beschränkt den Präsidenten fast vollständig auf die Funktionen des obersten Repräsentanten des Staates“ (ebd.). Und darum sei er ,tatsächlich keine wichtige Figur im politischen Kräftefeld der Bundesrepublik. Seine Autorität ruht nicht auf Macht, sondern auf der Art und Weise, wie er den gesamten Staat nach innen und außen vertritt" (283).

Hans-Peter Schwarz wird noch deutlicher: Der Bundespräsident könne mit seinem „repräsentativen Staatsamt“ gegenüber der Regierung genauso wenig bewegen, ,wie das ein Monarch in den meisten der noch verbliebenen konstitutionellen Monarchien vermag“ (2012: 300). Jedenfalls habe kein Amtsinhaber den ,großen Gang der Entwicklung“ verändert, geschweige denn es versucht: „Die Geschichte der Bundesrepublik wäre wahrscheinlich nicht anders verlaufen, wenn statt des nach den Art. 54-61 GG in Verbindung mit Art. 68 GG tätigen Bundespräsidenten im Jahresrhythmus der jeweilige Präsident des Bundesrats die entsprechenden Funktionen wahrgenommen hätte“ (298). Insofern kann man sich mit Klaus von Beyme in der Tat die Frage stellen, „ob es nicht systemgerechter gewesen wäre, auf ein repräsentatives Staatsoberhaupt gänzlich zu verzichten“ (2004: 304). Die institutionellen Kompetenzen des Präsidenten interpretiert von Beyme sehr restriktiv und in der Konnotation mit Distanz. Das materielle Prüfungsrecht nach Art. 82 GG sei 
Der Bundespräsident: Monarchisches Artefakt oder politisches Verfassungsorgan?

beispielsweise ,in gewisser Weise systemwidrig“, ein „Zopf aus der Zeit der konstitutionellen Monarchie (306). Die politische Bedeutung des Amtes erschließe sich weit mehr durch seine repräsentative Funktion als durch institutionelle Kompetenzen. „Und wo er über Handlungsspielräume verfügt“, sekundiert Frank Decker (2011: 331), sei er ,gehalten, diese nicht zu überdehnen und gegen den Willen der übrigen Staatsorgane auszuüben.“

Als Repräsentant der „Einheit des Staates“ und als Integrationsfigur agiert der Präsident in dieser Lesart zuallererst ,als eine Art Ersatzmonarch“ (Hesse/Ellwein 2012: 454). „Hier ist er frei, kann Populäres oder öffentlich kaum Bestreitbares moralisch anmahnen, empfehlen oder fordern, ohne für eine Verwirklichung verantwortlich zu sein. Er vermag auf diese Weise allgemeinen Stimmungen Ausdruck zu geben, Popularität zu gewinnen und die öffentliche Meinung zu beeinflussen“" (Rudzio 2011: 313). Allerdings setze das eine durch Überparteilichkeit in Fragen der Tagespolitik weitgehend durch Zurückhaltung gekennzeichnete Amtsführung voraus. Tatsächlich sei es „fast allen Bundespräsidenten“ gelungen, ,parteiübergreifende Zustimmung als ein nur dem , Ganzen' verpflichtetes Staatsoberhaupt zu finden“ (Jochum 2000: 46), obwohl sie vor ihrer Wahl durchaus gestandene Parteipolitiker waren. Umgekehrt, so Stefan Marschall, sei es „,verbreiteter Konsens in der Bundesrepublik, die Person und das Amt aus dem parteipolitischen Streit herauszuhalten. Dieser Konsens sollte freilich von den Amtsinhabern selbst nicht herausgefordert werden“ (2007: 193). Letzten Endes zeige sich die Funktion eines nicht-regierenden Staatsoberhauptes in der „Ergänzung von Elementen, die der sonstigen Politik abgehen. Zu diesen Elementen zählen insbesondere Überparteilichkeit, Langfristorientierung, Erklärung der 'großen Zusammenhänge', Vermittlung von Ziel und Richtung, Betonung des Beständigen inmitten hektischen Wandels" (Jochum 2000: 62).

Wozu also ein Bundespräsident? Notwendig sei das Amt nicht, gibt Hesse zu (so auch Rausch 1984: 115 ff.), aber im Hinblick auf ,auf einige wichtige Formalien“ doch ,wünschenswert“" (455). Sowohl der Regierungschef als auch der Parlamentspräsident seien letztlich zu ,parteiergreifender Aktivität gezwungen“, während nur das Staatsoberhaupt ,allgemeinen Ansprüchen zu genügen vermag“"(Hesse/Ellwein 2012: 455 f.). „Eines zu haben, bietet freilich drei Vorteile“, findet Werner Patzelt. Neben der Reservefunktion und der im Prüfungsrecht zutage tretenden Rechtswahrung ,mag es in symbolischen und emotionalen Tiefenschichten des Politischen jenseits des tagespolitischen Streits integrierend wirken“ (2005: 293). Frank Decker bedient sich des schon von Michael Jochum vorgebrachten Kompensationsarguments und attestiert dem Amt im Hinblick auf seine Repräsentations- und Integrationsfunktionen eine „entlastende Wirkung“, zumal eine „wie immer geartete Se- 
mipräsidentialisierung“ nicht zu befürchten sei (2011: 340): „Indem es die Basis der Repräsentation verbreitert, trägt es dazu dabei, die Legitimationsgrundlagen der Politik und des Staates zu stärken. Weshalb sollte die Republik auf diesen Beitrag, den alle bisherigen Bundespräsidenten auf ihre Art geleistet haben, ohne triftigen Grund verzichten?"“(ebd.: 341)

Doch offenbar gibt es zwischen einem konstitutionellen Monarchen und dem Staatsoberhaupt einer parlamentarischen Republik doch mehr Unterschiede als nur das „Selektionssystem“ (Kaltefleiter 1970: 23). In der Monarchie ist es die Krone, nicht ihr Träger, die die „Einheit der Nation“ symbolisiert (ebd.: 26). Insofern ist für die „Integration“ des Volkes schon Genüge getan, wenn sich die Monarchen auf den Vollzug tradierter politischer Zeremonien beschränken und ansonsten die „Untertanen“ an ihrem privaten Glück teilhaben lassen: Jubiläen, Geburtstage, Hochzeiten und natürlich eine erfolgreiche Fortpflanzung. ,Ruck-Reden” (Herzog) oder Forderungen nach „Vorfahrt für Arbeit“ (Köhler) wären hier nicht nur ein Fauxpas, sondern Anlass für veritable Verfassungskrisen. Dagegen erwartet die Republik von ihrem Staatsoberhaupt durchaus eine politische Rolle, zum einen, weil es hier keinen öffentlichen Anspruch auf das Privatleben eines gewählten Bürgers gibt und sinnvollerweise auch nicht geben kann; zum anderen, weil sich ein Wahlamt nicht allein durch seine schiere Existenz, sondern immer auch das Handeln seines Trägers zu rechtfertigen hat.

Wo die Grenzen einer öffentlichen, politischen Rolle des Bundespräsidenten liegen, lässt sich nicht a priori bestimmen. Aber die Politikwissenschaft hat sich die Definition der konstitutionellen Bedeutung des Bundespräsidenten samt einer entsprechend angemessenen Amtsführung unnötig schwer gemacht, indem sie die „Repräsentations-” und „Integrationsfunktion“ von der vielleicht einzig belastbaren Verfassungsfunktion getrennt hat: der Reservegewalt. Das führt zur diffusen und zuweilen auch „sakral“ (Schwarz 2012: 300) anmutenden Aufladung des Amtes, die als „Funktionen“ auf eine Überforderung der Amtsinhaber hinauslaufen. Denn am Ende muss immer offen bleiben, was unter der Fähigkeit zur Integration eigentlich verstanden werden kann. Politische Identitäten sind soziale Konstrukte, deren fortlaufende Erneuerung nicht allein durch politische Akteure und Institutionen gewährleistet werden kann, schon gar nicht durch eine einzige Person, sei sie auch Akteur und Institution in einem.

Stattdessen könnte der Bundespräsident als politischer Akteur begriffen werden, der eigene Interessen und Ziele im öffentlichen Diskurs verfolgen kann, zum Beispiel Popularität erlangen, Themen aufgreifen und setzen, überhaupt „erhört“ zu werden. Aber entscheidend bleibt, dass ihn sein öffentliches Wirken nicht daran hindert, im Krisenfall tatsächlich integrierend zu wirken, und zwar zwischen den 
Der Bundespräsident: Monarchisches Artefakt oder politisches Verfassungsorgan?

vom Volk gewählten Parteien, die allen Differenzen zum Trotz eine handlungsfähige Regierung zu bilden haben. Das muss im Übrigen auch nicht immer eine monarchische Zurückhaltung erfordern, sondern kann in einer auszumalenden historischen Situation durchaus öffentliche Kritik, Beistand oder „Parteinahme“ verlangen.

\section{Welche Macht der Amtsinhaber ausüben könnte: Das Gewaltenteilungs- paradigma}

Ingeborg Maus (2008: 11) macht den Idealtyp einer parlamentarischen Demokratie an zwei Merkmalen fest: einer vertikalen Gewaltenteilung zwischen ,gesetzgebender Souveränität und gesetzanwendenden Staatsapparaten" einerseits sowie einer direkten, vertikalen Legitimationskette zwischen Volk, Parlament und Regierung andererseits, die eine ungeteilte Volkssouveränität gewährleisten soll. Ihr Gegenmodell ist die amerikanische Unionsverfassung, die auf einer horizontalen Gewaltenteilung samt Souveränitätsteilung beruht: „Alle ,Gewalten' sind an der Ausübung der Souveränitätsfunktion beteiligt und treten zur Legislative in Konkurrenz, so daß die teilsouveränen Gewalten sich gegenseitig kontrollieren“" (2008: 11). Diese Gegenüberstellung verdeutlicht, was das Regierungssystem des Grundgesetzes nicht ist: eine in konsequenter Reinform institutionalisierte parlamentarische Demokratie. Die parlamentarische Architektur wird vielmehr durch horizontale Checks-and-Ballances-Elemente ergänzt oder - je nach Standpunkt - verfremdet. Dazu zählen in erster Linie die überaus starke, weil zur Normenkontrolle berufene, Verfassungsgerichtsbarkeit sowie das Bundesratsmodell, durch das die Exekutiven der Länder als Vetospieler im Gesetzgebungsprozess auftreten können. Das deutsche Regierungssystem zeichnet sich also durch einen „Verfassungseklektizismus“ (ebd.: 44) aus, der als „Politikverflechtung“ (Scharpf/Reissert/Schnabel 1976) oder als „Strukturbruch“ (Lehmbruch 1998) der „Verhandelnden Wettbewerbsdemokratie“" (Korte/Fröhlich 2009: 75-81) seine politikwissenschaftliche Aufarbeitung erfahren hat.

Das Regierungssystem des Grundgesetzes wird in der Literatur nur dann als ein „rein parlamentarisches“ (Hartmann/Kempf 2011: 91) oder „,konsequent“ (Rudizio 2011:308) parlamentarisches System bezeichnet, wenn der Bundespräsident in den Mittelpunkt rückt. Hier setzt die Kritik am Westminster-Paradigma an, zumal dieses nicht recht erklären kann, warum die Verfassungsgeber dem Staatsoberhaupt einerseits Entscheidungskompetenzen übertragen, andererseits aber erwarten, dass er sie gar nicht nutzt. Die Frage erübrigt sich, wenn man sich „ein Stück weit von der 'reinen' Lehre des Parlamentarismus verabschiedet und diesen als eine Form 
institutionalisierter und flexibler Gewaltenteilung sowie komplexer Repräsentation interpretiert" (Lhotta 2012: 134). Schließlich müsse eine verfassungs- und systemgerechte Interpretation des Präsidentenamtes der horizontalen Gewaltenkontrolle die gleiche Bedeutung beimessen, wie sie oft nur den parlamentarischen Verfassungselementen zuteil wird. Dann würde ersichtlich, dass der Bundespräsident „Bestandteil eines tief gestaffelten Systems von checks and balances“ sei, ,dem sich parlamentarische Mehrheiten in der Bundesrepublik Deutschland gegenüber sehen“ (2012: $141 \mathrm{f}$.).

Roland Lhotta greift damit einen in der Staatsrechtslehre entwickelten Argumentationsstrang auf, demzufolge dem Bundespräsidentenamt im Gewaltenteilungsgefüge keine eindeutige Funktion zukomme. Es sei vielmehr „komplementär zu allen übrigen Verfassungsorganen angelegt“, aber „nicht im Sinne einer Ersatzfunktion, sondern aus einer eigenen Verfassungsposition heraus ergänzend und unterstützend“" (Jekewitz 1984: Art. 54, zit. nach Lhotta 2012: 137). Der Präsident kann vor allem in zwei Bereiche eingreifen, die nach rein parlamentarischer Interpretation allein dem Parlament obliegen sollten: in die Regierungsbildung nach Art. 63 und 68 GG sowie in die Gesetzgebung durch sein Prüfungsrecht nach Art. 82 GG. Diese institutionellen Möglichkeiten machen ihn aber auch im Rahmen des Gewaltenteilungsparadigmas keinesfalls zu dem „Hüter der Verfassung“. Selbst wenn man den von Carl Schmitt geprägten und durch dessen Antiparlamentarismus und Antipluralismus kontaminierten Begriff verwenden möchte, wäre er zuallererst für das Bundesverfassungsgericht zutreffend. Dabei hat schon Kaltefleiter (1970: $208 \mathrm{ff}$.) darauf hingewiesen, dass letztendlich alle Verfassungsorgane die Verfassungsordnung zu schützen haben und es im extremsten aller Ausnahmefälle die Bürger selbst sind, die über ihr Widerstandsrecht für ihre Verfassung einstehen müssen.

Dennoch kommt dem Staatsoberhaupt im Gewaltenteilungsparadigma eine durch die Verfassung privilegierte Position zu, die es ihm erlaube, ,an der Ausübung der Staatsgewalt in funktionaler und gewaltenteiliger Verschränkung mit anderen Organen“ (Nettesheim 2005: 1054 f.) teilzunehmen. Diese Verschränkung sei „Ausdruck der Idee der gemischten Verfassung, in der Organe verschiedenen Zuschnitts die Ausübung der Staatsgewalt zugleich fördern und kontrollieren“ (ebd.). Woran aber wäre die eigenständige Rolle des Bundespräsidenten im Mobile sich gegenseitig kontrollierender Verfassungsorgane in der Praxis festzumachen? Vor allem an seinem materiellen Prüfungs- und Ausfertigungsverweigerungsrecht am Ende des Gesetzgebungsverfahrens. Es öffne dem Präsidenten ein „Portal zu einer Performanz als (fallabhängiger) Vetospieler“ (Lhotta 2012: 134). Oft werde übersehen, sekundiert Marcus Höreth (2012: 7), „dass der Bundespräsident formell am Gesetzgebungsprozess beteiligt ist und somit die Kriterien eines vollwertigen Veto- 
Der Bundespräsident: Monarchisches Artefakt oder politisches Verfassungsorgan?

spielers nach Tsebelis (2002) erfüllt"“. Wenn dem so ist, dann drängt sich eine Anschlussfrage auf: „Ab wann bzw. welcher Machtausstattung des Präsidenten wird ein parlamentarisches System in die Richtung eines präsidentiellen bzw. semi-präsidentiellen Systems verschoben? Die Möglichkeit jedenfalls, ein von der Parlamentsmehrheit getragenes Gesetz nicht auszufertigen und damit faktisch über ein (nicht überstimmbares) Veto zu verfügen, scheint hier eine der entscheidenden Variablen zu sein." (Lhotta 2012: 137) Wenn die Amtsinhaber ihr Prüfungsrecht in Zukunft expansiver nutzen sollten, könnte eine ,sektorale antimajoritäre Hybridisierung“" (ders. 2008: 121) des parlamentarischen Systems die Folge sein.

Anlass für diese Überlegungen ist die Präsidentschaft Horst Köhlers, der im Jahr 2006 zwei Gesetze der Großen Koalition nicht ausfertigte: das „Gesetz zur Neuregelung der Flugsicherung“ sowie das „Gesetz zur Verbesserung der gesundheitsbezogenen Verbraucherinformation". In beiden Fällen nennt der Präsident materielle Verfassungsverstöße als Gründe für sein „Veto“(Rütters 2011: 873). Allerdings erfüllen weder die von Köhler noch die vier von seinen Vorgängern nicht ausgefertigten Gesetze die Kriterien einer politischen Schlüsselentscheidung: große Reichweite der Betroffenen, Intensität der Regulierung und großes Konfliktpotenzial im politischen Wettbewerb (Beyme 1997: 66 f.) Hinzu kommt die geringe Zahl der Fälle: Zwischen 1949 und 2009 wurden ganze acht von 7.037 Gesetzen von den Bundespräsidenten nicht ausgefertigt. Angesichts einer Vetofrequenz von 0,1 Prozent und der geringen politischen Bedeutung der beanstandeten Gesetze kann man den Vertretern des Gewaltenteilungsparadigmas also durchaus entgegenhalten, dass die Bundespräsidenten bisher, ,weder als besonders aktive und für den politischen Entscheidungsprozess relevante 'Vetospieler'“ agierten, „noch verfolgten sie mit Hilfe der Veto-Option erkennbare eigenständige politisch-programmatische Ziele“. Eine Präsidentialisierung, so Rütters weiter, ließe sich ,allen Ernstes aus der bisherigen Anwendung der Ausfertigungsverweigerung weder ableiten noch ablesen noch extrapolieren" (2011: $875 \mathrm{f}$.).

Der Gegensatz der beiden Positionen ergibt sich aus ihren unterschiedlichen Herangehensweisen: Das „Vetospieler-Argument“ Lhottas ist ein theoretisches, aus der Gewaltenteilungslogik der Verfassung destilliertes Argument, das die Potenziale des Amtes auszuloten versucht, während Rütters, der Westminster-Logik folgend, die konstitutionelle Bedeutung des Präsidenten aus der bisherigen Amtspraxis ableitet. Marcus Höreth (2012) unternimmt den Versuch einer Vermittlung, indem er die institutionellen Möglichkeiten und die bisherige Praxis der Gesetzesausfertigung in einen politischen Kontext stellt. Vier der acht Ausfertigungsverweigerungen betrafen Gesetze einer Großen Koalition, deren Gesetzgebung darüber hinaus, so rechnet Höreth vor, überdurchschnittlich oft durch das Bundesverfassungsgericht 
annulliert worden sei, allerdings erst nach ihrer jeweiligen Regierungszeit. Offensichtlich gingen übergroße parlamentarische Mehrheiten mit einer geringeren verfassungsrechtlichen Sensibilität einher, weil eine uneinige Opposition nicht immer das notwendige Quorum für eine abstrakte Normenkontrolle aufzubringen vermag. Damit unterliege die Gesetzgebung großer Koalitionen einer verringerten verfassungsrechtlichen Kontrolle, was mit dem „Leitbild des demokratischen Verfassungsstaates" nicht zu vereinbaren sei. Hier kommt nun das Staatsoberhaupt ins Spiel: „Als fallabhängiger Vetospieler im Gesetzgebungsprozess wäre der Bundespräsident in der Lage, für die Dauer einer Großen Koalition die oben festgestellte reduzierte verfassungsrechtliche Kontrolldichte dadurch zu erhöhen, dass er sein Prüfungsrecht expansiver wahrnimmt als zu Zeiten einer kleinen Koalitionen und bei Zweifeln an der Verfassungsmäßigkeit eines ihm vorgelegten Gesetzes die Ausfertigung verweigert" (Höreth 2012: 13). Als Folge erwartet Höreth einen disziplinierenden Effekt: „Wenn zukünftig Große Koalitionen zum gängigen Koalitionsformat werden [...], wird der Bundespräsident stärker als je zuvor in die Rolle eines nicht immer einfach zu absorbierenden Vetospielers in der Gesetzgebung hineinwachsen. Dies wird nötig sein, um die regierende Große Koalition zu größerer verfassungsrechtlicher Sorgfalt zu ermutigen; oder dort, wo es nötig ist, den regelmäBigen Einsatz des BVerfG zu ermöglichen“" (Höreth 2012: 18).

Höreths „Mithüter-Funktion“ macht die konstitutionelle Bedeutung des Bundespräsidenten und die „Angemessenheit“ seiner Amtsführung von politischen Kräfteverhältnissen und historischen Kontexten abhängig. Sie ähnelt damit im Ansatz der „Vorbeugefunktion“ nach Xuewu Gu (1999), durch die dem normativen, aber auch statischen Funktionalismus des Westminster-Paradigmas eine ,prozessualdynamische" Sichtweise" entgegenstellt werden soll, indem sie die binäre Unterscheidung zwischen Normal- und Krisenfall überbrückt (763). Man dürfe nicht übersehen, „daß Funktionserwartungen oder Funktionsstörungen des Parlaments und/oder der Regierung sehr oft durch antizipierendes und konfliktabbauendes Handeln, ja manchmal auch durch geschicktes Drohen von Staatsmännern, also durch einen dynamischen politischen Prozeß vorgebeugt werden kann. Gerade aus diesem Vorbeugungsprozeß ergibt sich für den Bundespräsidenten, der von der Verfassung mit einem Gesetzprüfungsrecht (Art. 82), Parlamentsauflösungsrecht (Art. 63, 68) und Gesetzgebungsnotstandsrecht ausgestattet wird, eine ,Vorbeugefunktion', die er jeder Zeit ausüben kann und muß“" (ebd.: 764).

Um eine eigenständige und wirksame Rolle unter den Verfassungsorganen einnehmen zu können, muss der Bundespräsident seine verfassungsrechtlichen Möglichkeiten also gar nicht ausschöpfen. Entscheidend ist, dass er es könnte, und dass er seine Bereitschaft glaubhaft macht, es im Zweifelsfall auch zu tun. Er „könnte“ 
Der Bundespräsident: Monarchisches Artefakt oder politisches Verfassungsorgan?

schon während des parlamentarischen Entscheidungsprozesses den Kanzler auf seine Zweifel an einem Gesetz aufmerksam machen oder Bedenken gegen die Ernennung eines Ministers oder Beamten vortragen: „In diesem Sinne kann der Bundespräsident nicht nur politische Entscheidungen verhindern, sondern auch mitgestalten" (Gu 1999: 765). Dies ist auch deshalb eine gewagte These, weil zum einen offen bleibt, angesichts des Forschungsstandes offen bleiben muss, ob die Bundespräsidenten ihrer „Vorbeugefunktion“ tatsächlich nachzukommen versuchen, und ob sich Kanzler und Parlament zum anderen davon wirklich beeindrucken lassen. Im Grunde genommen kombiniert Gu den Konjunktiv des Gewaltenteilungsparadigmas mit dem Imperativ des Westminster-Paradigmas (Letzteres mit umgekehrten Vorzeichen): Weil die Präsidenten eine Vorbeugefunktion ausüben könnten, sollten sie es auch tun. Mehr noch: Gu stellt der Reserve- und Repräsentationsfunktion in Anlehnung an Eschenburg (1963: 649 f.) eine dritte Funktion zur Seite, in der Erwartung, dass sie im Regelfall auch ausgeübt wird, denn andernfalls läge ja ein prinzipielles Funktionsversagen vor. Das ist solange kein echter ,prozessualdynamischer“ Ansatz, solange die Ausübung der „Vorbeugefunktion“ nicht auch nachgewiesen werden kann.

Das verfassungsrechtliche Potenzial für eine „critical juncture“ im Hinblick auf die Normen einer angemessenen Amtsführung liegt vermutlich gar nicht in den Prüfungskompetenzen des Bundespräsidenten, die ja schließlich keinen genuin politischen Ermessensspielraum bieten. Bei der Regierungsbildung ist das aber sehr wohl der Fall, vorausgesetzt die bisher gekannte Stabilität der parlamentarischen Mehrheitsverhältnisse wäre in Zukunft nur noch eine Erinnerung an die „gute alte Zeit“. Wolfgang Rudzio hat das gesamte Machtpotenzial des Präsidenten im Falle einer Regierungskrise zusammengefasst: „Der Bundespräsident erschiene dann als zentraler Akteur. Vorstellbar würde - bei kombiniertem Einsatz seiner Möglichkeiten zu Kanzlerernennung, Parlamentsauflösung und zum Gesetzgebungsnotstand - quasi ein Präsidialregime, wenngleich deutlich abgeschwächt und zeitlich begrenzt" (2011: 312).

Dabei muss man gar nicht den Teufel eines Gesetzgebungsnotstandes an die Wand malen, um das Szenario für eine Neubewertung der konstitutionellen Bedeutung des Bundespräsidenten zu entwerfen. Dazu reicht schon eine neue Unübersichtlichkeit in den parlamentarischen Mehrheitsverhältnissen, wie sie Karl-Rudolf Korte (2013) beschrieben hat: ein Bundestag, vielleicht mit sechs oder sieben Parteien, in dem eine Koalitionsbildung entlang der tradierten Lager nicht möglich ist und in dem sich Union oder SPD einer Großen Koalition verweigern. Wenn eine Neuwahl an dieser Konstellation nichts ändert bzw. nach menschlichem Ermessen nichts ändern würde, läge die Regierungsbildung in den Händen des Bundespräsi- 
denten. Er könnte eine Minderheitsregierung ernennen oder eine Koalition zwischen jenen Parteien vermitteln, die ein solches Bündnis bisher ausgeschlossen haben. Vielleicht ist es tatsächlich nur die Autorität des Bundespräsidenten, der die Parteien von den Koalitionsversprechungen gegenüber ihren Wählern entbinden kann, um der Staatsräson willen - eine Mehrheitsregierung zu bilden, sei es in Form einer Ampel-, einer Jamaika- oder doch einer Großen Koalition.

All das ließe sich rein theoretisch noch unter die Reservefunktion des Staatsoberhauptes subsumieren, die ,den gestörten Mechanismus des parlamentarischen Systems wieder in Ordnung" setzen soll (Kaltefleiter 1970: 33). Aber wäre eine solche Regierung, insbesondere im Falle eines Minderheitenkabinetts, in den Augen von Öffentlichkeit und Opposition nicht auch seine Regierung? Wäre er nicht ihr Schirmherr, und zwar im engeren Sinne des Wortes als ihr Protektor? Welche Konsequenzen hätte das für seine „Symbol-“, „Repräsentations-“ oder „Integrationsfunktion"? Gewiss ist das alles spekulativ, zeigt aber, dass sowohl die konstitutionelle Bedeutung des Bundespräsidenten als auch eine entsprechend angemessene Amtsführung keinesfalls statisch sind.

\section{Wie das Amt ausgeübt wird: Ein Desiderat der politikwissenschaftlichen Forschung}

Wozu gibt es einen Bundespräsidenten? Der vorangegangene Überblick über die Argumentationsstränge in der politik- und staatswissenschaftlichen Forschung hat gezeigt, dass es an Vorstellungen über seine Funktionen nicht mangelt: Neben der Reserve- und Notariatsfunktion finden sich Symbol-, Repräsentations- und Integrationsfunktionen, noch dazu Vorbeuge-, Kontroll- und Wächterfunktionen. Zum Teil weisen die verschiedenen Rollendefinitionen gemeinsame Schnittmengen auf, zum Teil setzen sie eigene Akzente. Vereinfacht kann man zwischen jenen Rollendefinitionen unterscheiden, die herausstellen, wie die Bundespräsidenten ihr Amt angesichts der Funktionslogik des parlamentarischen Regierungssystems führen sollten (Westminster-Paradigma), und jenen, die aufzeigen, wie sie ihr Amt führen könnten, um ihrer Rolle im Gefüge sich gegenseitig kontrollierender Verfassungsorgane gerecht zu werden (Gewaltenteilungsparadigma).

Wie das Amt aber tatsächlich ausgeübt wird, und zwar auch in den für die Öffentlichkeit nur schwer einsehbaren Winkeln des Regierungssystems, ist ein bisher erstaunlich selten bearbeitetes Forschungsfeld. Doch solange wir nicht wissen, was die Bundespräsidenten in ihrem Arbeitsalltag tatsächlich tun, muss jede Antwort auf die Frage, wozu es sie gibt, lückenhaft ausfallen. Forschungsbedarf besteht vor allem in den folgenden Bereichen: 
Der Bundespräsident: Monarchisches Artefakt oder politisches Verfassungsorgan?

- Außenpolitik: Die völkerrechtliche Vertretung der Bundesrepublik gehört zu den wenigen konkreten, im Grundgesetz genannten Aufgaben des Bundespräsidenten. 60 Prozent seiner Zeit widme er der Außenpolitik, sagte Christian Wulff über seinen Arbeitsalltag als Präsident, ${ }^{3}$ und man kann annehmen, dass es bei anderen Präsidenten nicht viel weniger war bzw. ist. Doch für was müssen sie ihre Zeit konkret verwenden? Absolvieren sie nur Protokolle oder spielen sie, wenn schon keine eigenständige, so doch eine eigene außenpolitische Rolle im Einvernehmen mit der Bundesregierung? Können die Präsidenten Aufgaben übernehmen, die ihnen Kanzler und Außenminister aus guten Gründen überlassen?

- Verhältnis zu Parlament und Regierung: Wie eng sind die Kontakte zu Parlamentariern, Parteien und Regierungsmitgliedern im Arbeitsalltag der Präsidenten? Treten sie hier wirklich als Ratgeber, Mahner und Ermunterer auf? Und lassen sich Fälle identifizieren, die zeigen, dass die Präsidenten tatsächlich Einfluss auf die Meinungsbildung, vielleicht sogar auf konkrete Entscheidungen von Regierung und Parlament auszuüben vermögen? Entsprechende empirische Erkenntnisse - und eben nicht nur Anekdoten aus der biografischen Literatur wären deshalb so bedeutsam, weil nur sie Aufschluss darüber geben können, ob die „Auctoritas“(Eschenburg 1963: 649) oder „Vorbeugemacht“(Gu 1999) mehr sind als nur gern weitererzählte Legenden der Regierungslehre.

- Öffentlichkeitsarbeit und öffentliche Wirkung: Es wäre unrealistisch anzunehmen, die Bundespräsidenten erhielten automatisch qua Amt große mediale Aufmerksamkeit. Jeder politische Akteur muss sich seine Presse-Artikel und Sendeminuten hart erarbeiten. Über die Praktiken und Kalküle ihrer medialen Inszenierung und Wortpolitik ist indes kaum etwas bekannt. Eine andere Frage ist, wie erfolgreich sie dabei sind. Wolfgang Jäger glaubte zu Beginn der 1990er Jahre noch, die Mediendemokratie werde das öffentliche Gewicht der Bundespräsidenten erhöhen (1994: 182), während Hans-Peter Schwarz 18 Jahre später eine ,zunehmende Bedeutungslosigkeit“ (2012: 305) ausgemacht haben will. Wer recht hat, lässt sich mit Blick auf den Forschungstand nicht sagen. Für eine Bewertung seiner „Repräsentations- und Integrationsfunktion“ wäre es aber durchaus bedeutsam zu wissen, welchen Stellenwert präsidiale Reden in der Medienberichterstattung einnehmen und ob sie bis in das Bewusstsein einer breiten Öffentlichkeit vordringen.

Profunde empirische Analysen der Amtsführung, insbesondere hinter den medialen und zeremoniellen Kulissen, könnten zudem helfen, dem so emsigen wie auch auf

3 Vergleiche welt.de vom 11.12.2010, www.welt.de/politik/deutschland/article11552919/Wulff-istDeutschlands-heimlicher-Aussenminister.html (Stand: 4.11.2013). 
eigentümliche Art ahistorischen „Funktionen-Sammeln“ eine alternative Perspektive auf den Bundespräsidenten entgegenzustellen. Anstatt jenseits seiner verfassungsmäßigen Aufgaben nach „Funktionen“ zu suchen, die ja auch immer ein „Sollen“, wenn nicht gar ein „Müssen“ konstatieren, ließe sich das Amt des Bundespräsidenten dann besser über seine Optionen charakterisieren. Der deutsche Bundespräsident bekleidet ein institutionell schwaches, aber auch überaus unabhängiges Amt, das ihn jenseits seiner notariellen Aufgaben kaum zu etwas verpflichtet (Patzelt 2005). Aber er besitzt je nach historischen Herausforderungen und politischen Kräfteverhältnissen unterschiedliche, auch unterschiedlich große Möglichkeiten einer angemessenen Amtsführung. Auf welche Weise, unter welchen Bedingungen und ob überhaupt sich die Amtsinhaber all den oben genannten Funktionsfragmenten widmen, widmen sollen und können, lässt sich somit weder allein aus dem Verfassungstext noch allein aus der äußeren Anschauung der bisherigen Amtspraxis erschließen. Dazu bedürfte es systematischer empirischer Forschung, die eine Präsidentschaft in ihren jeweiligen politisch-historischen Kontext einordnet.

\section{Literatur}

Benda, Ernst/Maihofer, Werner/Vogel, Hans-Jochen (Hrsg.), 1983: Handbuch des Verfassungsrechts der Bundesrepublik Deutschland, Berlin/New York.

Beyme, Klaus von, 1997: Der Gesetzgeber. Der Bundestag als Entscheidungszentrum, Wiesbaden.

Beyme, Klaus von, 2004: Das politische System der Bundesrepublik Deutschland. Eine Einführung, Wiesbaden (10. Aufl.).

Decker, Frank, 2011: Regieren im „Parteienbundesstaat“. Zur Architektur der deutschen Politik, Wiesbaden.

Eschenburg, Theodor, 1963: Staat und Gesellschaft in Deutschland, Stuttgart.

Grunden, Timo, 2013: Wozu ein Bundespräsident? Amt und Amtsführung des deutschen Staatsoberhauptes in der staatswissenschaftlichen Forschung, in: Regierungsforschung.de, http://www.regierungsforschung.de/dx/public/article.html? id $=252$ (Stand: 29.11 .2013 ).

Gu, Xuewu, 1999: Die Vorbeugefunktion des Bundespräsidenten, in: Zeitschrift für Parlamentsfragen 30 (3), 761-771.

Hartmann, Jürgen/Kempf, Udo, 2011: Staatsoberhäupter in der Demokratie, Wiesbaden.

Hesse, Joachim Jens/Ellwein, Thomas, 2012: Das Regierungssystem der Bundesrepublik Deutschland, Baden-Baden (10. Aufl.). 
Der Bundespräsident: Monarchisches Artefakt oder politisches Verfassungsorgan?

Höreth, Marcus, 2008: Das Amt des Bundespräsidenten und sein Prüfungsrecht, in: Aus Politik und Zeitgeschichte (16), 32-38.

Höreth, Marcus, 2012: Grüß-August, Staatsnotar oder Vetospieler? Zur Bestimmung der Rolle des Bundespräsidenten im Regierungssystem. Papier für den DVPW Kongress, Universität Tübingen, 25.-29. September 2012, http:// www.dvpw.de/fileadmin/docs/Kongress2012/Paperroom/2012Regieren-H \%F6reth-2.pdf (Stand: 4.11.2013)

Jäger, Wolfgang, 1994: Wer regiert die Deutschen? Innenansichten der Parteiendemokratie, Zürich.

Jekewitz, Jürgen, 1984: Der Bundespräsident, Art. 54, in: Richard Bäumlin u. a. (Hrsg.), Kommentar zum Grundgesetz für die Bundesrepublik Deutschland, Neuwied/Darmstadt.

Jochum, Michael, 2000: Worte als Taten. Der Bundespräsident im demokratischen Prozess der Bundesrepublik Deutschland, Gütersloh.

Kaltefleiter, Werner, 1970: Die Funktionen des Staatsoberhauptes in der parlamentarischen Demokratie, Köln/Opladen.

Korte, Karl-Rudolf, 2013: Kanzlermacher Gauck, in: Süddeutsche Zeitung, 19.7.2013, http://www.sueddeutsche.de/politik/koalitionen-nach-bundestags wahl-kanzlermacher-gauck-1.1725383 (Stand: 4.11.2013).

Korte, Karl-Rudolf/Fröhlich, Manuel, 2009: Politik und Regieren in Deutschland. Strukturen, Prozesse, Entscheidungen, Paderborn u. a. (3. Aufl.).

Lehmbruch, Gerhard, 1998: Parteienwettbewerb im Bundesstaat, Stuttgart (2. Aufl.).

Lhotta, Roland, 2008: Der Bundespräsident als „Außerparlamentarische Opposition"? Überlegungen zur Gewaltenteilung und Typologisierung des parlamentarischen Regierungssystems, in: Zeitschrift für Parlamentsfragen 39 (1), 119-133. Lhotta, Roland, 2012: Der Bundespräsident als Vetospieler? Gewaltenteilung und komplexe Repräsentation im deutschen Parlamentarismus, in: Robert Chr. van Ooyen/Martin H. W. Möllers (Hrsg.), Der Bundespräsident im politischen System, Wiesbaden,131-142.

Marschall, Stefan, 2007: Das politische System Deutschlands, Konstanz.

Maus, Ingeborg, 2008: Über Volkssouveränität. Elemente einer Demokratietheorie, Frankfurt a. M.

Nettesheim, Martin, 2005: Amt und Stellung des Bundespräsidenten in der grundgesetzlichen Demokratie, in: Josef Isensee/Paul Kirchhof (Hrsg.), Demokratie - Bundesorgane, Bd. 3, Heidelberg, 1031-1072 (3. Aufl.).

Niclauß, Karlheinz, 1998: Der Weg zum Grundgesetz. Demokratiegründung in Westdeutschland 1945-1949, Paderborn u. a. 
Otto, Volker, 1971: Das Staatsverständnis des Parlamentarischen Rates. Ein Beitrag zur Entstehungsgeschichte des Grundgesetzes für die Bundesrepublik Deutschland, Bonn.

Patzelt, Werner J., 2005: Der Bundespräsident, in: Oscar W. Gabriel/Everhard Holtmann (Hrsg.), Handbuch Politisches System der Bundesrepublik Deutschland, 291-308 (3. Aufl.).

Rausch, Heinz, 1984: Der Bundespräsident - zugleich eine Darstellung des Staatsoberhauptes in Deutschland seit 1919, München.

Rudzio, Wolfgang, 2011: Das politische System der Bundesrepublik Deutschland, Wiesbaden (8. Aufl.).

Rütters, Peter, 2011: Worüber wir reden, wenn wir über den Bundespräsidenten reden. Oder: ... auf dem Weg zur Präsidentialisierung des Regierungssystems?, in: Zeitschrift für Parlamentfragen 42 (4), 883-885.

Scharpf, Fritz W./Reissert, Bernd/Schnabel, Fritz (Hrsg.), 1976: Politikverflechtung. Theorie und Empirie des kooperativen Föderalismus in der Bundesrepublik, Kronberg.

Schmidt, Manfred G., 2007: Das politische System Deutschlands, München.

Schwarz, Hans-Peter, 2012: Von Heuss bis Köhler. Die Entwicklung des Amtes im Vergleich der Amtsinhaber, in: Robert Chr. van Ooyen/Martin H. W. Möllers (Hrsg.), Der Bundespräsident im politischen System, Wiesbaden, 285-306.

Sontheimer, Kurt, 1995: Grundzüge des politischen Systems der neuen Bundesrepublik Deutschland, München/Zürich (16. Aufl.).

Strohmeier, Gerd, 2008: Der Bundespräsident: Was er kann, darf und muss bzw. könnte, dürfte und müsste, in: Zeitschrift für Politik 55 (2), 155-198.

Tsebelis, George, 2002: Veto Players. How Political Institutions Work, New York.

Korrespondenzanschrift:

Dr. Timo Grunden

Institut für Politikwissenschaft

Justus-Liebig-Universität Gießen

Karl-Gloeckner-Straße 21 E

35394 Gießen

E-Mail: Timo.Grunden@sowi.uni-giessen.de 